\title{
Carotenoid-Chlorophyll Coupling and Fluorescence Quenching Correlate with Protein Packing Density in Grana-Thylakoids
}

\author{
Christoph-Peter Holleboom, ${ }^{\dagger}$ Sunny Yoo, ${ }^{\ddagger}$ Pen-Nan Liao, ${ }^{\dagger}$ Ian Compton, ${ }^{\S}$ Winfried Haase, $"$
} Helmut Kirchhoff, $*, \S$ and Peter Jomo Walla $*, \dagger, \perp$

\footnotetext{
${ }^{\dagger}$ Institute of Physical and Theoretical Chemistry, Department of Biophysical Chemistry, Technische Universität Braunschweig, Hans-Sommer-Straße 10, 38106 Braunschweig, Germany

¥Sungkyunkwan University, Department of Energy Science, Suwon 440-746, Korea.

${ }^{\S}$ Institute of Biological Chemistry, Washington State University, P.O. Box 646340, Pullman, Washington 99164, United States

"Department of Structural Biology, Max Planck Institute of Biophysics, Max-von-Laue-Straße 3, 60438 Frankfurt am Main, Germany

${ }^{\perp}$ AG Biomolecular Spectroscopy and Single-Molecule Detection, Max-Planck-Institute for Biophysical Chemistry, Am Faßberg 11, 37077 Göttingen, Germany.
}

ABSTRACT: The regulation of light-harvesting in photosynthesis under conditions of varying solar light irradiation is essential for the survival and fitness of plants and algae. It has been proposed that rearrangements of protein distribution in the stacked grana region of thylakoid membranes connected to changes in the electronic pigment-interaction play a key role for this regulation. In particular, carotenoid-chlorophyll interactions seem to be crucial for the downregulation of photosynthetic light-harvesting. So far, it has been difficult to determine the influence of the dense protein packing found in native photosynthetic membrane on these interactions. We investigated the changes of the electronic couplings between carotenoids and chlorophylls and the quenching in grana thylakoids of varying protein packing density by two-photon spectroscopy,

\section{BBY Membrane Protein Density}
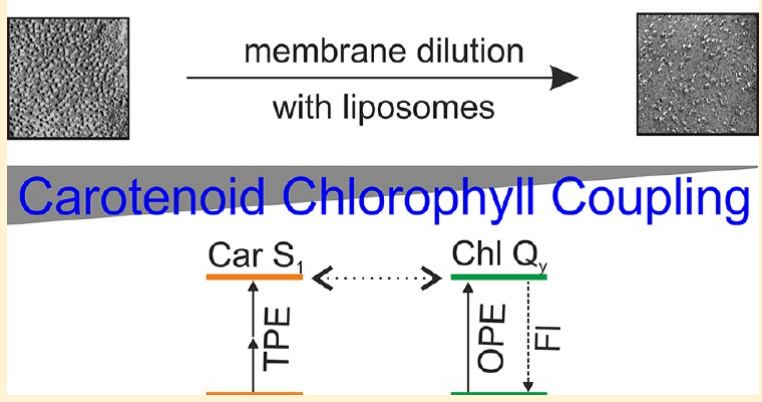
conventional chlorophyll fluorometry, low-temperature fluorescence spectroscopy, and electron micrographs of freeze-fracture membranes. We observed an increasing carotenoid-chlorophyll coupling and fluorescence quenching with increasing packing density. Simultaneously, the antennas size and excitonic connectivity of Photosystem II increased with increasing quenching and carotenoid-chlorophyll coupling whereas isolated, decoupled LHCII trimers decreased. Two distinct quenching data regimes could be identified that show up at different protein packing densities. In the regime corresponding to higher protein packing densities, quenching is strongly correlated to carotenoid-chlorophyll interactions whereas in the second regime, a weak correlation is apparent with low protein packing densities. Native membranes are in the strong-coupling data regime. Consequently, PSII and LHCII in grana membranes of plants are already quenched by protein crowding. We concluded that this ensures efficient electronic connection of all pigment-protein complexes for intermolecular energy transfer to the reaction centers and allows simultaneously sensitive regulation of light harvesting by only small changes in the protein packaging.

\section{INTRODUCTION}

Photosynthesis in higher plants and algae is initiated by the harvesting of sunlight through a series of pigment-protein complexes located in the thylakoid membranes of chloroplasts. Starting from these light harvesting complexes (LHCs), the absorbed energy is efficiently transferred from one complex to the other. These transfer processes end up in the photosystem reaction centers where the energy is converted into redox energy. However, plants are exposed to changing light intensities on a daily basis that can vary over several orders of magnitude. ${ }^{1}$ Thus, a regulation mechanism is necessary that assures effective usage of sunlight energy under low-light conditions but avoids photo-oxidative damage to the reaction centers or other parts of the photosynthetic apparatus if the absorbed light exceeds its capacity. A main mechanism that evolved to minimize photo-oxidative damage in plants is called high-energy quenching $(\mathrm{qE})$. $\mathrm{qE}$ enables the dissipation of excess energy as harmless heat. This important regulation of energy utilization is essential for the survival and fitness of plants. ${ }^{2}$ However, the precise mechanism of $\mathrm{qE}$ and in particular the exact site of energy dissipation in the photosynthetic apparatus is still a matter of intensive discussions.

Photosynthetic pigments embedded in the LHCs are crucial for light harvesting. Besides chlorophylls that collect the major fraction of sunlight, carotenoids also take part in light

Special Issue: Rienk van Grondelle Festschrift

Received: November 30, 2012

Revised: February 8, 2013

Published: February 12, 2013 
harvesting. They show absorption bands in the blue spectral region that are caused by a strong optical transition to their second excited singlet state $\left(S_{2}\right)$. As one-photon excitation of the first excited state $\left(S_{1}\right)$ is optically forbidden, this state does not directly contribute to the harvesting process. A large part of the energy absorbed by carotenoids is transferred to chlorophylls directly from the $S_{2}$ or after internal conversion via the $S_{1}$ state. In addition to this function in light harvesting, carotenoids also participate in various types of photoprotection including excess energy dissipation. Whether the changes in electronic interactions between carotenoids and chlorophylls and the corresponding regulation process are originated by conformational rearrangements inside pigment-protein complexes $^{3-5}$ or by interactions at their periphery ${ }^{6}$ is still debated, just as the question of which complexes are effectively involved. ${ }^{3-8}$ Also, different mechanisms of how the carotenoid $\mathrm{S}_{1}$ state is involved electronically in $\mathrm{qE}$ have been discussed. Important examples are (i) a $\mathrm{Chl} \rightarrow$ Car $\mathrm{S}_{1}$ energy transfer followed by fast internal conversion to the Car $S_{0}$ state, ${ }^{9,10}$ (ii) a Car $S_{1} \rightarrow$ Chl electron transfer forming a transient chargeseparated state, ${ }^{11-13}$ and (iii) excitonic Chl $Q_{y} \leftrightarrow$ Car $S_{1}$ interactions causing chlorophyll lifetime reduction. ${ }^{14-16}$ The Chl-Car interactions underlying these models have all been observed using different carotenoid-phthalocyanine dyads, providing evidence that they are potentially all possible. ${ }^{17-19}$ Two-photon excitation enables directly addressing the optically forbidden Car $S_{1}$ state. It was shown that the determination of the electronic coupling strength between Car $S_{1}$ and $C h l Q_{y}$ is possible by this approach. A comparison of fluorescence observed after direct chlorophyll one-photon excitation $\left(\mathrm{Fl}^{\mathrm{OPE}}\right)$ with the chlorophyll fluorescence observed after previous twophoton excitation $\left(\mathrm{Fl}^{\mathrm{TPE}}\right)$ of the optically forbidden carotenoid $S_{1}$ states directly provided quantitative information about the actual electronic carotenoid-chlorophyll coupling in a sample: ${ }^{14}$

$$
\Phi_{\text {coupling }}^{\mathrm{CarS}_{1}-\mathrm{Chl}}(\mathrm{t})=\frac{\mathrm{Fl}^{\mathrm{TPE}}(t)}{\mathrm{Fl}^{\mathrm{OPE}}(t)}
$$

The most abundant pigment-protein complex in higher plants and algae is the light harvesting complex II (LHCII). Some studies suggested that LHCII also plays a central role in the regulation of light harvesting. ${ }^{5,20}$ Like other LHCs, it is an integral membrane protein in thylakoids, but in contrast to other LHCs, it is organized as a trimer. ${ }^{21}$ Furthermore, it is estimated that LHCII, which accounts for approximately $30 \%$ of all thylakoid membrane proteins in plants, binds about half of the total chlorophyll in chloroplasts. ${ }^{22}$ By crystal structure analysis, it was shown that one LHCII monomer contains 8 Chlorophyll a, 6 Chlorophyll b, and 4 carotenoids. ${ }^{23,24}$ In the thylakoid membranes, several LHCs and therefore a large number of pigments are linked to photosystem reaction centers. Thus, light harvesting energy transfer is not only necessary between pigments inside a complex but also between LHCs in the thylakoid membrane. Because such energy transfer processes are strongly dependent on the distance, membrane organization, and especially packing of LHCs, the protein density is likely to have a significant impact on the efficiency of photosynthetic light-harvesting. Actually, there is a quite high protein density in thylakoid membranes, in particular, in the stacked grana thylakoid domain where about $70 \%$ of the membrane area belongs to proteins. ${ }^{25}$ Protein packing is likely an important factor in the regulation of light-harvesting as low protein densities might result in disconnection of lightharvesting complexes from the reaction center and high protein densities might lead to energy dissipating interactions. It was already shown that the chlorophyll fluorescence lifetime and thus also the fluorescence quenching of LHCs incorporated in liposomes are affected by the density of LHCs in membranes. ${ }^{4}$ Furthermore, research on isolated LHCII trimers demonstrated a dependency between aggregation of LHCII and energy quenching. ${ }^{6,10,26}$ Additionally, it was found that aggregation of LHCII trimers in vitro also causes an increase of the abovementioned coupling parameter. ${ }^{27}$ However, it is difficult to assess how much isolated LHCII and models of aggregation quenching really reflect the in vivo situation. A much better approach to gain insight into the influence of the protein packing density in thylakoid membranes is varying the packing density in native grana thylakoids by fusion with liposomes harboring a lipid composition of natural thylakoids. By using such membranes, it was demonstrated that the light harvesting behavior is significantly influenced by different protein packing densities. ${ }^{28}$ However, so far it is not known whether these changes in light-harvesting efficiency are also connected to an electronic coupling between carotenoids and chlorophylls or if they are just a result of changes in protein packing densities. This question is essential for gaining more insight into the causality of the molecular processes, for example, whether fluctuations in protein densities are actually inducing conformational changes within pigment-protein complexes or are directly causing new carotenoid-chlorophyll interactions at the periphery of membrane proteins. Therefore, we investigated the dependencies of carotenoid-chlorophyll electronic interactions as well as fluorescence quenching on the protein packing in grana membranes by two-photon spectroscopy and compared the results to other functional parameters such as antenna size, the connectivity of photosystem II, and LHCII coupling to PSII. We found clear correlations of electronic interactions, antenna size, the connectivity of Photosystem II, LHCII coupling, and the quenching with protein packing. The results indicated that depending on the excess in excitation energy, green photosynthetic organisms aim to connect electronically as many pigment-protein complexes as possible either to the reaction centers or to quenching centers that dissipate excess excitation energy by electronic carotenoidchlorophyll interactions. Our study indicated that quenching and Chl-Car coupling are not homogenously dependent on protein packing and that at least two distinct mechanisms of deactivation of electronic excited states exist.

\section{EXPERIMENTAL METHODS}

Preparation of Grana Membranes with Altered Protein Density and LHCII. Arabidopsis thaliana wild type plants were grown for 7 to 8 weeks at about $100 \mu \mathrm{mol}$ quanta $\mathrm{m}^{-2} \mathrm{~s}^{-1}$ and $9 \mathrm{~h}$ daylight. For thylakoid isolation, about $30 \mathrm{~g}$ of leaf material was blended in $330 \mathrm{mM}$ sorbitol, $50 \mathrm{mM}$ Hepes ( $\mathrm{pH} 7.5, \mathrm{KOH}), 2 \mathrm{mM}$ EDTA, $15 \mathrm{mM} \mathrm{NaCl}, 5 \mathrm{mM} \mathrm{MgCl}_{2}, 5$ $\mathrm{mM} \mathrm{CaCl} 2$, and $0.1 \%(\mathrm{w} / \mathrm{v})$ BSA filtered through 1 layer of Miracloth and 4 layers of muslin. Chloroplasts were obtained from the homogenate by pelleting them at $3000 \mathrm{~g}$. Chloroplasts were shocked in $50 \mathrm{mM}$ Hepes ( $\mathrm{pH} 7.5), 150 \mathrm{mM} \mathrm{NaCl}$, and 5 $\mathrm{mM} \mathrm{MgCl} 2$ for $2 \mathrm{~min}$ and the larger unsolubilized material was pelleted at $200 \mathrm{~g}$ for $1 \mathrm{~min}$. Intact thylakoids were isolated by centrifugation of the supernatant at $3000 \mathrm{~g}$ for $10 \mathrm{~min}$ and washed in $0.1 \mathrm{M}$ sorbitol, $50 \mathrm{mM}$ Hepes, $15 \mathrm{mM} \mathrm{NaCl}$, and 10 $\mathrm{mM} \mathrm{MgCl}$ (washing buffer). Grana membranes (BBY) were 

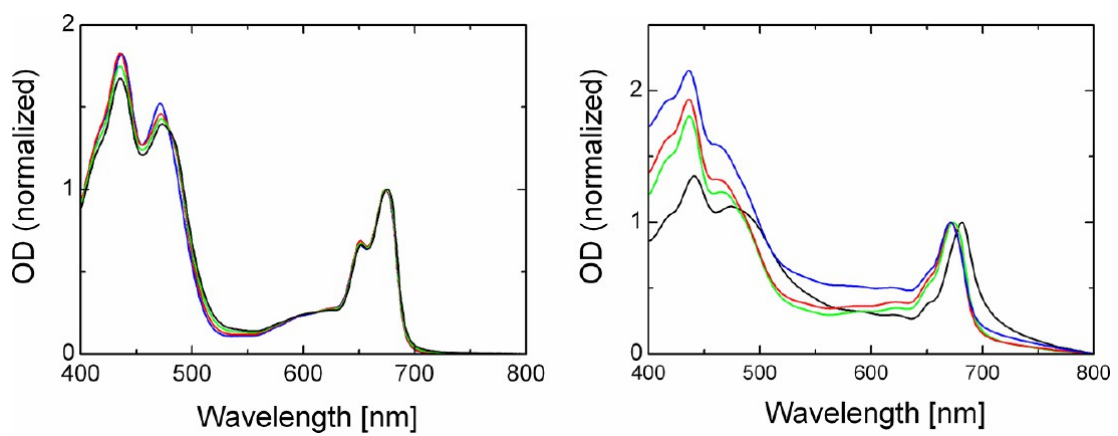

Figure 1. Absorption spectra. Left: LHCII in different aggregation states normalized to $Q_{y}$ maximum (blue, unquenched; red, green, black, increasing quenching). Right: grana membrane samples normalized to $Q_{y}$ maximum (black, unfused; green, red, blue, increasing fusion).

isolated from intact thylakoid membranes as described in ref 29. Chlorophyll concentrations were determined spectroscopically in $80 \%(\mathrm{v} / \mathrm{v})$ acetone. ${ }^{30}$ BBY membranes were fused with small unilamellar vesicles of the native grana thylakoid membrane lipid mixture as described by Haferkamp et al. ${ }^{31}$ The lipid/ chlorophyll stoichiometry of unfused and fused BBY membranes was determined by two different methods: Via twodimensional thin layer chromatography ${ }^{31}$ and by fatty acid quantification by gas chromatography.

Native LHCII samples of spinach were prepared as described previously. ${ }^{32}$ LHCII was diluted in a buffer solution of $50 \mathrm{mM}$ Tris, $\mathrm{pH} 7.5$, and $0.3 \%$ NG ( $n$-nonyl- $\beta$-D-glucopyranoside) and aggregated by detergent removal with SM-2 absorbent (BioRad) as described by Liao et al. ${ }^{27}$ Before coupling measurements, aggregated LHCII and grana membrane samples were diluted to similar OD. The OD values for the different series of measurements were adjusted between 0.11 and $0.37 \mathrm{~mm}^{-1}$ (at $\mathrm{Q}_{\mathrm{y}, \max }$ ).

Absorption and RT Fluorescence Spectra. Absorption spectra were recorded in a Lambda $25 \mathrm{UV} /$ Vis spectrometer (Perkin-Elmer). Fluorescence spectra were measured with a Varian Cary Eclipse fluorescence spectrometer after excitation with blue-green light of a wavelength area of $400-500 \mathrm{~nm}$. The absorption spectra of the LHCII as well as the grana membrane samples are displayed in Figure 1. The membrane spectra contain some features, such as the relatively strong red shift in the absorption spectrum of the undiluted grana membranes that can be attributed to scattering effects. The chlorophyll concentration of all samples within a series of measurement was adjusted to similar values. Please note, however, that the electronic coupling is not significantly influenced by the sample concentration as variations in the concentration are normalized in the calculation of $\Phi_{\text {coupling }}$ (see eq 1 and Bode et al. ${ }^{14}$ for details).

77 K Fluorescence Spectra. Low temperature (77 K) fluorescence spectra were recorded with a HoribaYvon Fluoromax-4 spectrofluorometer. The membranes were suspended in BBY measuring medium $(40 \mathrm{mM} \mathrm{KCl}, 7 \mathrm{mM}$ $\mathrm{MgCl}_{2}, 15 \mathrm{mM}$ MES $\mathrm{pH}$ 6.5) at a chlorophyll concentration of $\sim 3 \mu \mathrm{M}$, shock frozen in liquid nitrogen, and excited with 475 $\mathrm{nm}$ (band-pass $5 \mathrm{~nm}$ ). The emission bandwidth was $4 \mathrm{~nm}$. Four emission spectra/samples were averaged and corrected for the spectral response of the setup. For spectral deconvolution, these starting spectra were normalized in such a way that the area under the spectrum equals the measured $F_{m}$ value (see above for steady state $F_{m}$ measurements). The resulting spectra were fitted with five Gaussian curves, corresponding to free LHCII $\left(\mathrm{F}_{680}\right)$, CP43 $\left(\mathrm{F}_{685}\right)$, CP47 $\left(\mathrm{F}_{695}\right)$, aggregated LHCII
$\left(\mathrm{F}_{700}\right)$, and PSI plus satellite bands of the others $\left(\mathrm{F}_{730}\right)$ as described previously. ${ }^{28,33}$

Freeze Fracture Electron Microscopy. The membranes were freeze-fractured and examined by electron microscopy as described previosly. ${ }^{34}$ The membranes were placed in a copper sample holder, frozen to $-180{ }^{\circ} \mathrm{C}$ in liquefied ethane, and fractured in a BAF400T (BalTec, Principality Liechtenstein). Platinum/carbon replicas were imaged with an EM208S (FEI Co.).

Two-Photon Spectroscopy in Connection with PAM. Determination of the electronic coupling parameter was done as described previously. ${ }^{14}$ Briefly, a confocal setup was used that provided the possibility of measuring chlorophyll fluorescences $\mathrm{Fl}^{\mathrm{OPE}}$ and $\mathrm{Fl}^{\mathrm{TPE}}$ in the same sample at the same time. As a source of TPE light, an ultrafast laser system was used. The IR light was generated as an idler beam in an optical parametric amplifier OPA 9450 that was pumped by a combination of a Vitesse Duo and a RegA 9000 (all components from Coherent Inc.). The carotenoids were excited by $1188 \mathrm{~nm}$ light. To avoid chlorophyll one-photon excitation, the visible part of the OPA output was rejected by a heat mirror (L46-386, Edmund Optics) and a filter combination of 1100 and $900 \mathrm{~nm}$ long pass filters (FEL1100 and FEL900, Thorlabs). Fluorescence was detected for this excitation method by an ultrafast photodiode (designed by Prof. D. Schwarzer) and selectively amplified by a lock-in amplifier (EG\&G 5205). The lock-in was synchronized with a mechanical chopper positioned in the excitation beam. Control experiments determining dependencies of detected Signals on the excitation power were performed to verify the occurrence of two photon excitation within this method (data not shown). OPE and the appendant fluorescence detection was executed with a conventional PAM fluorometer (FMS1, Hansatech). The wavelength of the modulated excitation beam was $594 \mathrm{~nm}$.

\section{RESULTS}

Diluting the Protein Packing Density in Isolated Grana. For studying the impact of different protein packing densities on $\Phi_{\text {coupling, }}$ BBY membranes (BBY according to Berthold et $\mathrm{al}^{29}$ ) ) were fused with liposomes to incorporate additional lipids as described before. ${ }^{31}$ As unfused starting material, we used a BBY preparation that was heavily depleted in lipids. Lipid depletion is indicated by lipid analysis that showed a mol-lipid/mol-Chl ratio of only $\sim 0.5$, i.e., significantly lower than the ratio of 1.3 measured for BBY in previous studies. ${ }^{31,28}$ A consequence of the low lipid content in unfused BBY membranes, there is a drastically quenched chlorophyll fluorescence yield caused by protein overcrowding. ${ }^{28}$ The 
Table 1. Fluorescence and electronic coupling data of fused grana membranes and aggregated LHCII ${ }^{a}$

\begin{tabular}{|c|c|c|c|c|c|c|c|c|c|c|}
\hline \multirow[b]{2}{*}{ sample } & \multicolumn{5}{|c|}{ grana $\mathrm{BBY}$ membrane } & \multicolumn{5}{|c|}{ LHCII } \\
\hline & unfused & $\begin{array}{l}\text { fused } \\
\text { state } 1\end{array}$ & $\begin{array}{l}\text { fused } \\
\text { state } 2\end{array}$ & $\begin{array}{l}\text { fused } \\
\text { state } 3\end{array}$ & $\begin{array}{l}\text { fused } \\
\text { state } 4\end{array}$ & unquenched & $\begin{array}{l}\text { quenched } \\
\text { state } 1\end{array}$ & $\begin{array}{l}\text { quenched } \\
\text { state } 2\end{array}$ & $\begin{array}{l}\text { quenched } \\
\text { state } 3\end{array}$ & $\begin{array}{l}\text { quenched } \\
\text { state } 4\end{array}$ \\
\hline $\mathrm{F}^{\mathrm{OPE}}$ & 0.015 & 0.126 & 0.29 & 0.54 & 0.7614 & 1.0000 & 0.52 & 0.39 & 0.170 & 0.0577 \\
\hline \pm & 0.003 & 0.003 & 0.05 & 0.04 & 0.0009 & 0.0008 & 0.01 & 0.02 & 0.003 & 0.0008 \\
\hline $\mathrm{F}^{\mathrm{TPE}}$ & 0.04 & 0.26 & 0.29 & 0.42 & 0.522 & 1.000 & 0.60 & 0.48 & 0.31 & 0.14 \\
\hline \pm & 0.01 & 0.04 & 0.06 & 0.09 & 0.005 & 0.003 & 0.02 & 0.02 & 0.06 & 0.02 \\
\hline$\Phi_{\text {coupling }}$ & 2.6 & 2.1 & 0.97 & 0.8 & 0.685 & 1.000 & 1.14 & 1.23 & 1.8 & 2.5 \\
\hline \pm & 0.3 & 0.2 & 0.06 & 0.1 & 0.007 & 0.003 & 0.03 & 0.03 & 0.4 & 0.3 \\
\hline
\end{tabular}

${ }^{a}$ Data represent at least two independent measurements (LHCII quenched state 4 only one). LHCII unquenched sample was used as reference. All results were normalized to corresponding unquenched LHCII. Fluorescence values were corrected by an OD factor $\left(\Delta \mathrm{O} D=\mathrm{Q}_{\mathrm{y}, \max }-634 \mathrm{~nm}\right)$. Coupling parameters were calculated for each series of measurements separately and normalized and averaged afterwards.
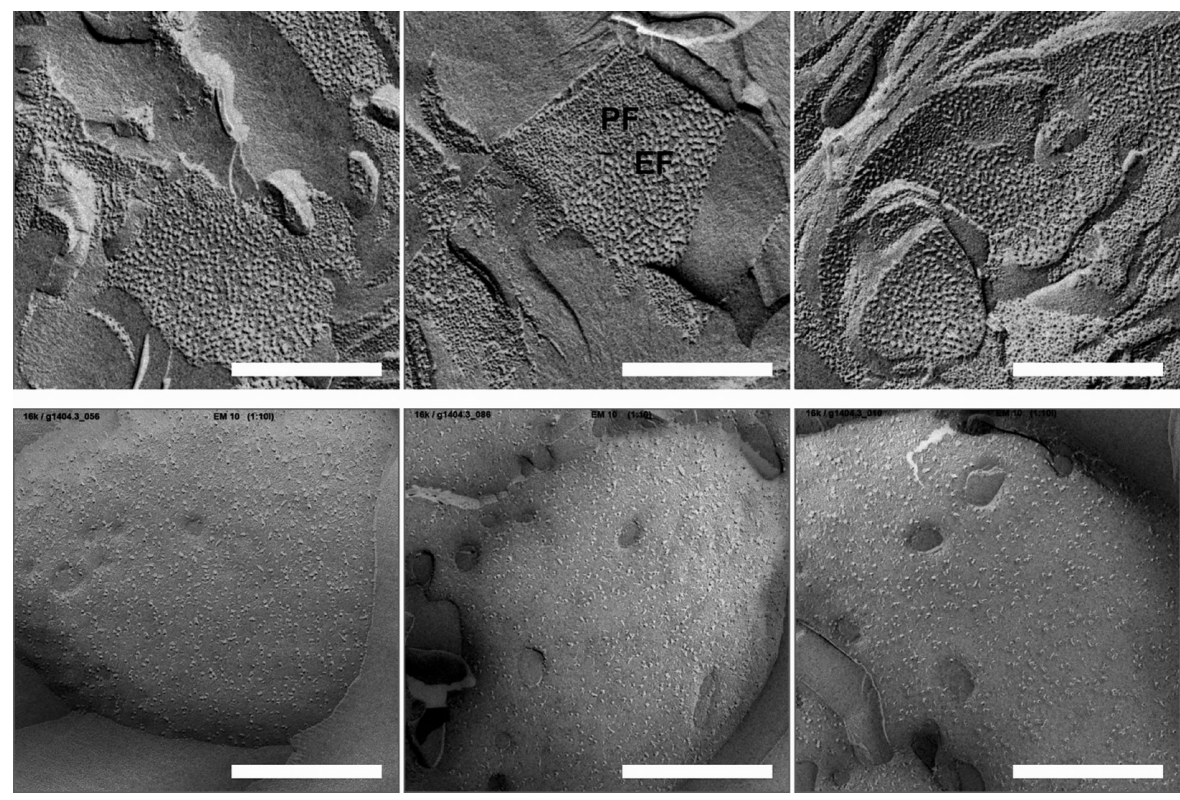

Figure 2. Electron micrographs of freeze-fractured grana BBY membranes (upper panel, unfused; lower panel, fused, additional lipid/chlorophyll $[\mathrm{mol} / \mathrm{mol}]=10$; scale bars $200 \mathrm{~nm}$ ). In freeze-fracture the membrane bilayer is split into two monolayers (exoplasmic fracture, EF and protoplasmic fracture, PF). Membrane integral protein complexes (PSII, LHCII) protrude as little knobs out of the monolayer plane (see figure). It is well established ${ }^{39}$ that the larger particles represent PSII (see, i.e., the EF face of the unfused BBY membrane) whereas the smaller protrusions represent LHCII (see, i.e., the PF face of the unfused BBY membrane). Note the significantly lower particle density after fusion with lipid liposomes (lower panel) compared to unfused BBY membranes (upper panel), indicating dilution of the protein density.

quenched state in unfused BBY is demonstrated by the very low $\mathrm{F}^{\mathrm{OPE}}$ (1.5\% relative to unquenched LHCII, Table 1$)$ and by the dominant $700 \mathrm{~nm}$ fluorescence band $\left(\mathrm{F}_{700}\right)$ in the $77 \mathrm{~K}$ emission spectra (Figure 3). $\mathrm{F}_{700}$ indicates quenching of LHCII fluorescence by aggregation ${ }^{35-38}$ that appears in overcrowded BBY membranes. ${ }^{28}$ Thus, the starting material in our examinations was grana membrane with an artificially high protein packing density. Starting from these overcrowded membranes, the protein packing density was diluted by fusion with lipid liposomes. The fused membranes have lower protein densities and a higher lipid content, but their lipid composition does not differ from unfused grana membranes because the BBY lipids were used for liposome preparation. ${ }^{28}$ Figure 2 shows freeze-fracture electron microscopy (EM) images of unfused (top) and fused (bottom) BBY membranes from equivalent samples as used in this study. The liposome-fused BBY had an additional lipid amount of $10 \mathrm{~mol}$ per mole of chlorophyll. The numerous protrusions seen in the freezefracture EM images represent membrane integral protein complexes (PSII, larger particles and LHCII, smaller particles $\left.^{39}\right)$. Comparison of the upper and the lower images in Figure 2 illustrates the much lower particle density in liposome-fused BBY indicative of a lower protein packing density.

Functional interactions between pigment protein complexes in unfused and fused BBY membranes were studied by lowtemperature fluorescence spectroscopy. Figure 3 shows chlorophyll emission spectra of unfused and various diluted membrane samples (added lipids/chlorophyll ratios 7.9, 10.9, and $23.3 \mathrm{~mol} / \mathrm{mol}$ ) at $77 \mathrm{~K}$. The spectra in A and C correspond roughly to the membrane situation shown in Figure 2. We deconvoluted the spectra into five emission bands as described previously. ${ }^{33,28}$ According to Haferkamp et al. ${ }^{28}$ and Andreeva et al., ${ }^{33}$ deconvolution yields information about free $\left(\mathrm{F}_{680}\right)$ and aggregated LHCII $\left(\mathrm{F}_{700}\right)$, the PSII core antenna proteins CP43 $\left(\mathrm{F}_{685}\right)$ and $\mathrm{CP} 47\left(\mathrm{~F}_{695}\right)$, LHCI, and finally PSI as well as higher vibrational bands of the previously mentioned components $\left(\mathrm{F}_{730}\right)$. One of the most prominent differences between the spectra of untreated and fused BBY is a strong increase of the amount of free, not aggregated, LHCII $\left(\mathrm{F}_{680}\right)$ in fused BBY that 


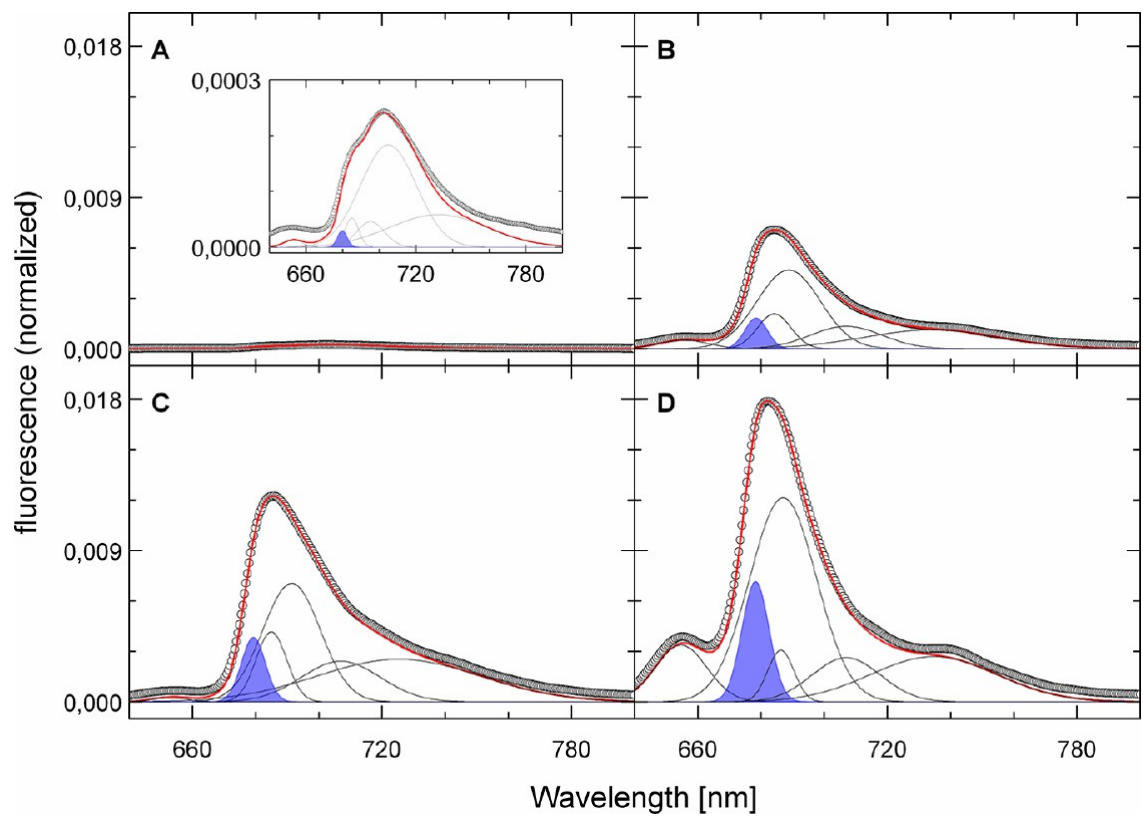

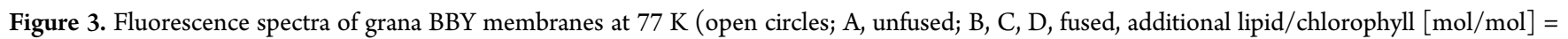
7.9, 10.9, 23.3). The area under the spectra was normalized to the measured $F_{\mathrm{m}}$ value. The red and black lines are fitted curves for analysis according to the multicomponent fit by Andreeva et al. ${ }^{33}$ The blue filled curve represents the $680 \mathrm{~nm}$ component (functionally uncoupled LHCII trimmers).

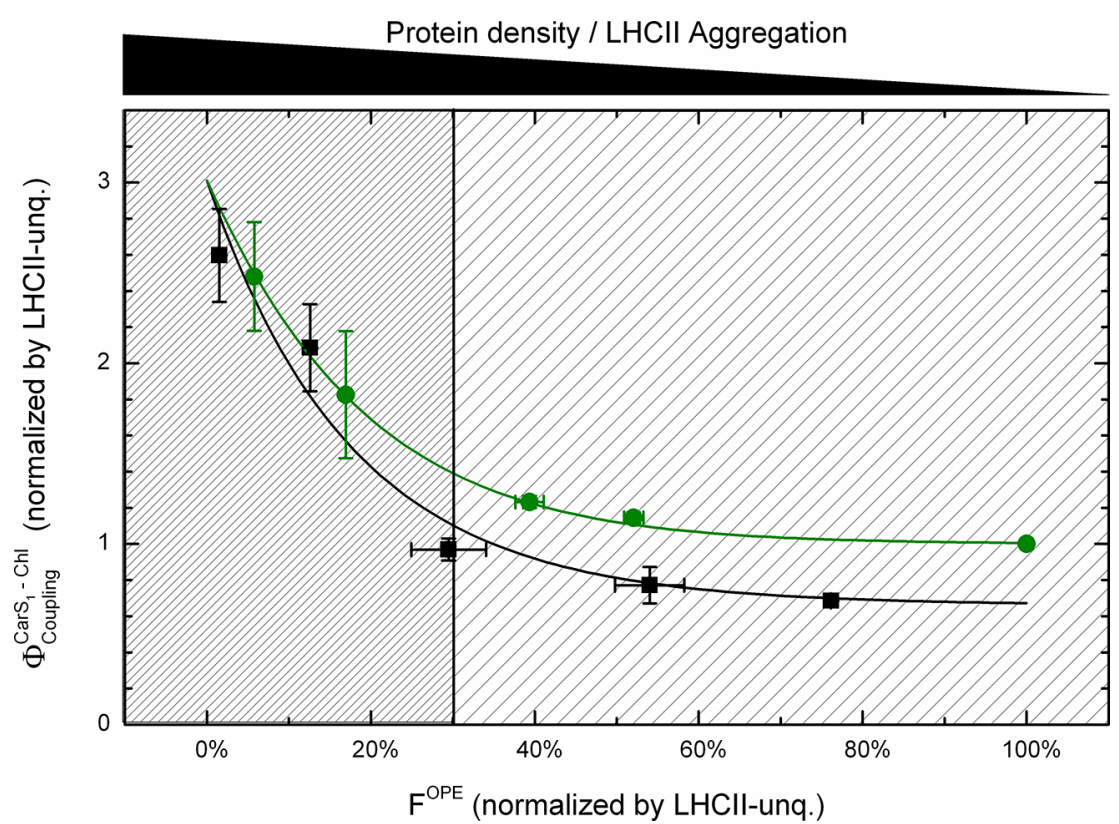

Figure 4. Electronic coupling dependence on effective fluorescence quenching: green circles, LHCII; black squares, grana membranes. For BBY membranes: the higher the $F^{\mathrm{OPE}}$ values, the higher the lipid/Chl ratio; i.e., points on the far left correspond to unfused BBY or strong aggregated LHCII, and points on the far right correspond to diluted membranes or nonaggregated LHCII. The vertical line indicates the transition between the strong-coupling and the weak-coupling data regime (see text for explanations). All data are normalized by unquenched LHCII $=1$.

grows stronger as more lipids are incorporated into the membrane. This effect was previously observed for such grana membranes. ${ }^{28}$ In summary, decreasing the protein density in overcrowded grana membranes leads to gradual functional uncoupling of LHCII from PSII which is indicated by increasing $\mathrm{F}^{\mathrm{OPE}}$ and $\mathrm{F}_{680}$.

Impact of Protein Density on $\boldsymbol{\Phi}_{\text {coupling }}$. Figure 4 and Table 1 show the influence of the protein packing density on the electronic carotenoid-chlorophyll coupling of a series of grana membranes with different protein densities. Constant $\mathrm{F}^{\mathrm{OPE}}$ values in the course of the measurement (variation less than 5\%) indicate that the samples are not photodamaged during the experiment (data not shown). Furthermore, the BBY samples are expected to be in the $F_{\mathrm{o}}$ state (oxidized primary quinone acceptor QA) because the samples were dark-adapted, the PAM measuring pulses are very weak (in the order of 0.001 $\mu$ mol quanta $\mathrm{m}^{-2} \mathrm{~s}^{-1}$ ) and the far-red TPE pulses $(1188 \mathrm{~nm}$ ) are not actinic. Figure 4 reveals a clear decrease in the coupling in BBY membranes with diluted protein packing densities that correlates with an increase in $\mathrm{F}^{\mathrm{OPE}}$. As previously described, the electronic coupling parameter $\Phi_{\text {coupling }}$ was calculated from the chlorophyll fluorescence intensities observed after alternating 
one- and two-photon excitation, $\mathrm{F}^{\mathrm{OPE}}$ and $\mathrm{F}^{\mathrm{TPE}}$, respectively. In addition, Figure 4 shows that the coupling of isolated LHCII complexes decreases correspondingly with decreasing aggregation and quenching, as previously observed. ${ }^{27}$ For a quantitative comparison of the couplings observed in LHCII with those in diluted grana membranes, all data were normalized to the corresponding $\mathrm{F}^{\mathrm{OPE}}$ value of unquenched LHCII (Figure 4, Table 1). For high $\mathrm{F}^{\mathrm{OPE}}$ values, this comparison provides evidence that the electronic coupling in grana membranes is lower (lower $\Phi_{\text {coupling }}$ ) than in pure LHCII. From Haferkamp et al., ${ }^{28}$ it is known that for added lipid/Chl ratios $>8$ all LHCII and PSII complexes are functionally separated by dilution with lipids. Thus, for the highest BBY $\mathrm{F}^{\mathrm{OPE}}$ value (added lipid/Chl $\sim 23$ ), the protein complexes are well separated and function like isolated entities. The $\sim 30 \%$ lower coupling in the maximal diluted BBY sample containing both PSII and LHCII compared to the unquenched isolated LHCII sample indicates less Car $\rightarrow$ Chl energy transfer via the forbidden Car $S_{1}$ state in PSII relative to LHCII. An explanation for the differences in the minimal $\Phi_{\text {coupling }}$ values between isolated LHCII and grana membranes could be reasoned by a certain amount of coupled Car-Chl pairs that are predominantly located in other parts of the PSII (not LHCII) and probably not taking part in fluorescence quenching or at least not that strongly.

Figure 4 reveals a quite heterogeneous correlation between $\Phi_{\text {coupling }}$ and chlorophyll fluorescence quenching (decreased $\mathrm{F}^{\mathrm{OPE}}$ ). From $0 \%$ to $\sim 30 \% \mathrm{~F}^{\mathrm{OPE}}$ (see vertical line that indicates the separation of two regimes), the coupling parameter is strongly correlated with $\mathrm{F}^{\mathrm{OPE}}$, whereas at values $>30 \% \mathrm{~F}^{\mathrm{OPE}}$, the correlation is weak; i.e., large changes in fluorescence quenching correspond to only small changes in $\Phi_{\text {coupling. }}$ In the following, we will name the first data regime ( $0 \%$ to $\sim 30 \%$ $\mathrm{F}^{\mathrm{OPE}}$, Figure 4 on the left) the strong-coupling regime and the second the one weak-coupling regime (Figure 4 on the right). For the weak-coupling regime, the BBY curve is parallel shifted to lower coupling values relative to isolated LHCII, most likely for the reason mentioned above. In the strong-coupling regime, the correlation between $\Phi_{\text {coupling }}$ and $\mathrm{F}^{\mathrm{OPE}}$ is indistinguishable between grana membranes and isolated LHCII at least within the error limits of the experiment. The missing difference between the two sample types in the strong-coupling data regime indicates that the coupling process(es) occurring here overcompensate the mechanism(s) that determine the coupling in the weak-coupling data regime. However, the good correlation between isolated LHCII and grana in the strongcoupling regime reveals that the quenching mechanism(s) realized in aggregated LHCII could be the dominating one(s) in grana membranes.

\section{DISCUSSION}

For studying aggregation-induced quenching, isolated LHCII was often regarded as a model system for NPQ mechanisms and indeed it shares many similarities with processes occurring in planta. ${ }^{10}$ For example, similar changes in the fluorescence lifetime or the fluorescence as well as absorption spectra are observed. However, it is obvious that the isolated LHCII model system has limitations, as it is hard to tell to what extent it really mimics crowding of pigment-protein complexes in the membrane. For example, the relative orientations of the pigment-protein complexes are quite arbitrary, leading to different surface interactions and different angles and distances between surface-exposed chlorophylls in adjacent LHCII trimers. ${ }^{40}$ In addition, missing lipid and membrane interactions could modify pigment interactions artificially in aggregated LHCII. Changing the protein density of native membranes by diluting with native lipid compositions to study aggregationinduced fluorescence quenching more closely resembles natural conditions. It has to be pointed out that chlorophyll fluorescence quenching as examined in this study is quite different from high-energy quenching in intact plants because the $\Delta \mathrm{pH}$ required for establishing $\mathrm{qE}$ cannot be formed in isolated grana membranes. Furthermore, the membranes were isolated from dark-adapted plants and therefore the $\mathrm{qE}$ activator zeaxanthin is absent. However, the similar correlation between chlorophyll fluorescence quenching and $\Phi_{\text {coupling }}$ of isolated LHCII and grana membranes (Figure 4) suggests that aggregation of LHCII mainly determines crowding-induced quenching in grana membranes. This is also in good agreement with findings gained for LHCs reconstituted in liposomes. A decrease of chlorophyll fluorescence lifetimes was found when the number of LHCs in the membrane was increased. ${ }^{4}$ Comparable to the results presented here that study indicated a correlation of chlorophyll fluorescence quenching in a membrane and the corresponding protein density.

Figure 5 summarizes the results of this study. Starting from highly quenched overcrowded grana with high $\Phi_{\text {coupling }}$ (Figure

Overcrowded Crowded

Figure 5. Schematic model of fused (diluted and crowded) and unfused (overcrowded) BBY membranes. LHCII trimers (green circles) and PSII complexes (gray) with reaction center dimers (red) are shown. Reduction of protein density leads to a decrease of PSII antenna size and fading excitonic coupling of the reaction centers. This is correlated with reduced electronic carotenoid-chlorophyll coupling (thinner black arrows).

5 , right), the addition of lipids leads to more native protein packing density with reduced coupling (Figure 5, middle). This moderate crowding is required to keep the efficiency of light harvesting of PSII by intermolecular energy transfer high. ${ }^{28}$ If membranes are more diluted (weak-coupling regime referring to Figure 4, Figure 5, left), electronic interactions between chlorophylls and carotenoids become uncoupled associated with functional disconnection between LHCII and PSII (high F680). The finding of weak-coupling and strong-coupling data regimes strongly indicates that quenching by carotenoidchlorophyll coupling requires a minimal protein packing density. For BBY membranes, it can be estimated that the threshold protein density between the two coupling data regimes is at added lipid/chlorophyll ratio of about 8 (the data point at which the vertical line differentiates the two regimes in Figure 4). As mentioned above, this is an interesting value because it is in a similar range where LHCII and PSII complexes are functionally separated. ${ }^{28}$ The observation that the strong-coupling data regime starts at a similar protein packing density where intercomplex energy transfer becomes significant is in line with carotenoids and chlorophylls involved in $\Phi_{\text {coupling }}$ that are localized at the protein periphery but could also be caused by protein structure changes due to packing density increase which effects pigments inside the protein. 


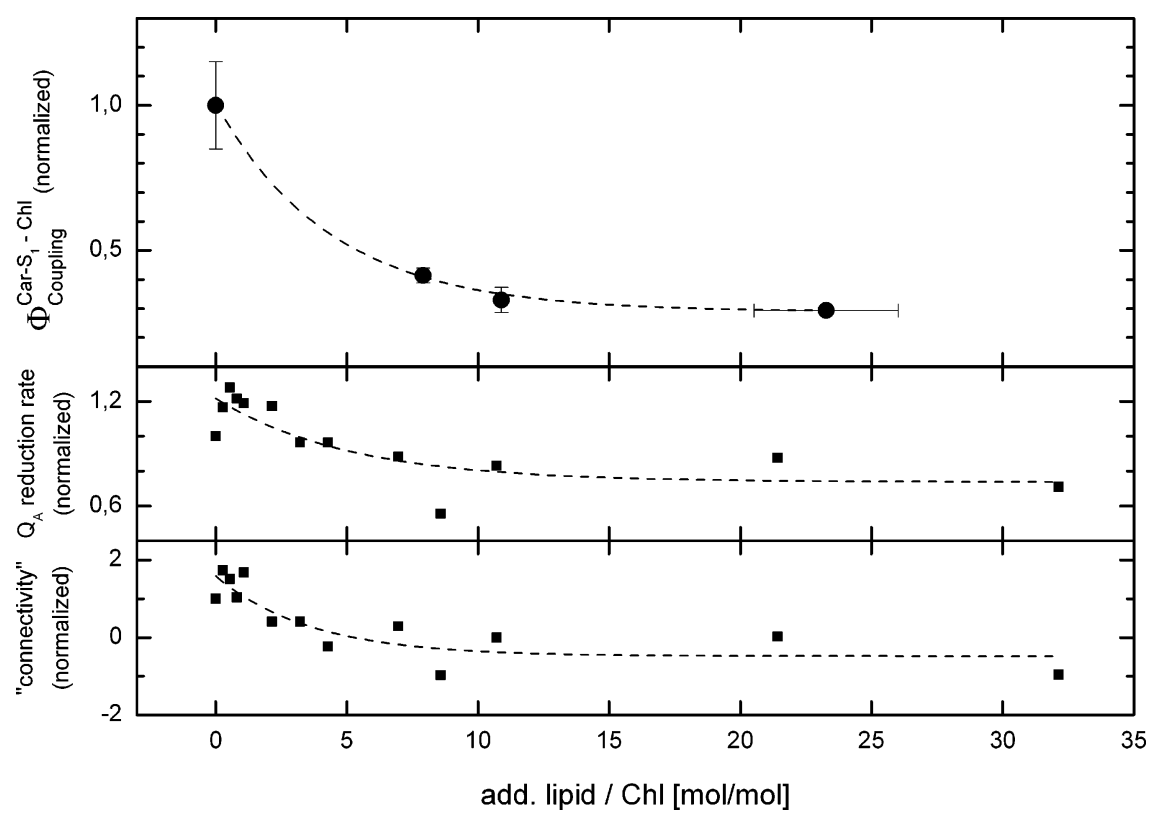

Figure 6. Carotenoid-chlorophyll coupling and protein packing density. Top: the electronic coupling of Car- $S_{1}$ state and chlorophyll $\mathrm{Q}_{y}$ state decreases with an increasing amount of incorporated lipids into the grana membrane. Middle and bottom: the $\mathrm{Q}_{\mathrm{A}}$ reduction rate and PSII connectivity data are taken from Haferkamp et al. ${ }^{28}$ to compare their correlation with the protein packing density (inversely correlated with the lipid/ Chl ratio).

The existence of a weak-coupling data regime in Figure 4 indicates that functional isolated proteins (either LHCII or LHCII and PSII in grana) can be quenched by aggregation (F $\mathrm{F}^{\mathrm{OPE}} 30-100 \%$ ) with less involvement of carotenoidchlorophyll coupling. This suggests that another $\Phi_{\text {coupling }}$ independent quenching mechanism is involved in the weakcoupling regime. At this point, we can only speculate about this mechanism but because it is dependent on protein (LHCII) aggregation, it could also involve chlorophyll-chlorophyll interactions. It was postulated that in native thylakoid membranes some part of the LHCII complexes form small aggregates that quench excitons by a chlorophyll-chlorophyll charge transfer process. ${ }^{41,7}$ This quenching type could be involved in the weak-coupling data regime. Noteworthy, this weak-coupling correlated quenching occurs in unphysiological diluted membranes ( $>8 \mathrm{~mol}$ lipids/mol Chl) because the lipid/ chlorophyll ratio in native grana is between 1 and $3 .^{28,42}$

As the lipid/chlorophyll ratio in grana membranes drops below 8, intermolecular energy transfer between LHCII-PSII and LHCII-LHCII is apparent that correlates with $\Phi_{\text {coupling. }}$ To demonstrate the correlation between electronic carotenoidchlorophyll coupling in more detail, we replotted the data from Haferkamp et al. ${ }^{28}$ in Figure 6. The extent of energy transfer in the PSII-LHCII antenna system was concluded from the apparent PSII antenna size (Figure 6 middle panel) and as the connectivity parameter (Figure 6, lower panel). The antenna size is a measure of the number of pigment-protein complexes or light-harvesting pigments that connect to a single PS II by excitation energy transfer. The PS II connectivity is a measure of how much one PSII is functionally connected to other PS II by intermolecular excitation energy transfer. The antenna size is derived from the $Q_{A}$ reduction rate that reflects the time an exciton needs to be transferred from the periphery of the antenna to the reaction center. In Figure 6, a direct comparison of the electronic carotenoid-chlorophyll coupling with the $Q_{A}$ reduction rate (reflecting the antennas size) as well as the PSII connectivity as observed in Haferkamp et al. ${ }^{28}$ are shown as a function of different values in added lipid per chlorophyll. It is obvious from Figure 6 that $\Phi_{\text {coupling }}$ and light-harvesting parameters of LHCII-PSII have a similar dependency on the lipid content in grana, i.e., lower protein density corresponds to less electronic carotenoid-chlorophyll coupling, less PSII connectivity, and a decreased size of the antenna system.

$\mathrm{F}^{\mathrm{OPE}}$ values of native membranes are expected to be in the strong-coupling data regime of Figure $4\left(<30 \% \mathrm{~F}^{\mathrm{OPE}}\right)$ because the lipid/Chl ratio in grana is well below 8 , i.e., the threshold value of the weak- to strong-coupling data regime. An important consequence of this fact is that relative to isolated nonaggregated LHCII, PSII, and LHCII in grana membranes in plants are already quenched to some extend by protein crowding. Although this seems surprising, it appears to be a price that has to be paid to ensure efficient intermolecular energy transfer, as demonstrated in Figure 6. Furthermore, in the strong-coupling regime, small changes in protein packing densities in grana have a strong impact on energy quenching mediated by changes in carotenoid-chlorophyll coupling (Figure 4). This is advantageous for regulation of light harvesting by PSII because reversible changes in protein packing density in grana could be an elegant way to control photoprotective energy dissipation. In this respect, recent findings of large-scale protein reorganizations in grana associated with high-energy quenching are interesting. ${ }^{43,44}$ These studies on intact thylakoid membranes show that the protein packing density in grana changes in the course of $\mathrm{qE}$ induction. Interestingly, these ultrastructural changes in grana depend on the presence of the PsbS protein, ${ }^{43}$ a key player for establishing $\mathrm{qE}^{45}$ Thus, a potential role of PsbS could be the control of protein packing. However, further studies are required to verify this possibility. It still remains difficult to elucidate the precise molecular mechanism of the quenching process or even to state at which site of the protein the carotenoid-chlorophyll coupling exactly occurs. Nevertheless, 
the clear correlation between the protein packing density and the fluorescence quenching as well the Car-Chl coupling provides evidence that there has to be at least one mechanism for $\mathrm{qE}$ that comprises some protein-protein or membraneprotein interactions. This can either lead to conformational changes within the proteins or by changes interactions at the periphery of the proteins or to both. Of course this does not exclude additional mechanisms such as conformational changes within individual proteins induced just by a $\mathrm{pH}$ gradient. A hint to a possible switch for $\mathrm{qE}$ is provided by recent studies on grana membranes similar to those used in this work. It was observed by resonance Raman spectroscopy that a bending of the neoxanthin is correlated with the crowding in thylakoid membranes. $^{28}$ The more the protein density is reduced the more this distortion fades. It can be assumed that this structural modification of neoxanthin causes changes in the protein which might be a trigger for high-energy quenching.

\section{CONCLUSIONS}

In summary, the data presented here show a clear decrease in electronic carotenoid-chlorophyll coupling, fluorescence quenching, and disconnection of photosynthetic pigmentprotein complexes within the membrane when the protein density in grana BBY membranes is decreased. In addition, these observations can be correlated with a concomitant decrease in antenna size and PSII connectivity, as reported previously. A direct comparison of these observations with the influence of LHCII aggregation on the electronic carotenoidchlorophyll coupling and fluorescence quenching supports again similarities between this in vitro model and the native membrane situation. Our data support a protein-crowdingdependent mechanism of $\mathrm{qE}$ that seeks to connect excitonically as many pigment-protein complexes as possible. Excess excitation energy is dissipated by electronic carotenoidchlorophyll interactions. Two distinct quenching data regimes could be identified that show up at different protein packing densities (Figure 4). In the dense regime, quenching is strongly correlated to carotenoid-chlorophyll interactions whereas this correlation is weak for the diluted situation. Native membranes are in the strong-coupling data regime. Consequently, relative to isolated nonaggregated LHCII, PSII, and LHCII in grana membranes of plants are already quenched by protein crowding. We conclude that this allows operation with efficient electronic connection of all pigment-protein complexes for intermolecular energy transfer to the reaction centers and simultaneously sensitive regulation of light harvesting by only small changes in the protein packaging.

\section{AUTHOR INFORMATION}

\section{Notes}

The authors declare no competing financial interest.

\section{ACKNOWLEDGMENTS}

H.K. is supported by the Washington State Agricultural Research Center (ARC), the National Science Foundation (MCB-1158571), and by the United States-Israel Binational Agricultural Research and Development Fund (US-4334-10). The Work is also financially supported by a Grant of the German Science Foundation (DFG, WA1305/3-2). S.Y. was supported by a global internship grant from the National Research Foundation of Korea (NRF) funded by the Korea government (MEST). Robert Yarbrough is acknowledged for proofreading the manuscript.

\section{REFERENCES}

(1) Demmig-Adams, B.; Adams, W. W. Science 2002, 298, 21492153.

(2) Kühlheim, C.; Agren, S.; Jansson, S. Science 2002, 297, 91-93.

(3) Ilioaia, C.; Johnson, M. P.; Horton, P.; Ruban, A. V. J. Biol. Chem. 2008, 283, 29505-29512.

(4) Moya, I.; Silvestri, M.; Vallon, O.; Cinque, G.; Bassi, R. Biochemistry 2001, 40, 12552-12561.

(5) Pascal, A. A.; Liu, Z.; Broess, K.; Oort, B. v.; Amerongen, H. v.; Wang, C.; Horton, P.; Robert, B.; Chang, W.; Ruban, A. Nature 2005, 436, 134-137.

(6) Barros, T.; Royant, A.; Standfuss, J.; Dreuw, A.; Kühlbrandt, W. EMBO J. 2009, 28, 298-306.

(7) Holzwarth, A. R.; Miloslavina, Y.; Nilkens, M.; Jahns, P. Chem. Phys. Lett. 2009, 483, 262-267.

(8) Mozzo, M.; Passarini, F.; Bassi, R.; van Amerongen, H.; Croce, R. Biochim. Biophys. Acta 2008, 1777, 1263-1267.

(9) Frank, H. A.; Cua, A.; Chynwat, V.; Young, A.; Gosztola, D.; Wasielewski, M. R. Photosynth. Res. 1994, 41, 389-395.

(10) Ruban, A. V.; Berera, R.; Ilioaia, C.; van Stokkum, I. H. M.; Kennis, J. T. M.; Pascal, A. A.; van Amerongen, H.; Horton, P.; van Grondelle, R. Nature 2007, 450, 575-579.

(11) Ahn, T. K.; Avenson, T. J.; Ballottari, M.; Cheng, Y.-C.; Niyogi, K. K.; Bassi, R.; Fleming, G. R. Science 2008, 320, 794-797.

(12) Avenson, T. J.; Ahn, T. K.; Zigmantas, D.; Niyogi, K. K.; Li, Z.; Ballottari, M.; Bassi, R.; Fleming, G. R. J. Biol. Chem. 2008, 283, 35503558.

(13) Holt, N. E.; Zigmantas, D.; Valkunas, L.; Li, X.-P.; Niyogi, K. K.; Fleming, G. R. Science 2005, 307, 433-436.

(14) Bode, S.; Quentmeier, C. C.; Liao, P.-N.; Hafi, N.; Barros, T.; Wilk, L.; Bittner, F.; Walla, P. J. Proc. Natl. Acad. Sci. U. S. A. 2009, 106, 12311-12316.

(15) Ma, Y. Z.; Holt, N. E.; Li, X. P.; Niyogi, K. K.; Fleming, G. R. Proc. Natl. Acad. Sci. U. S. A. 2003, 100, 4377-4382.

(16) van Amerongen, H.; van Grondelle, R. J. Phys. Chem. B 2000, 105, 604-617.

(17) Liao, P.-N.; Pillai, S.; Gust, D.; Moore, T. A.; Moore, A. L.; Walla, P. J. J. Phys. Chem. A 2011, 115, 4082-4091.

(18) Berera, R.; Herrero, C.; van Stokkum, I. H. M.; Vengris, M.; Kodis, G.; Palacios, R., E.; van Amerongen, H.; van Grondelle, R.; Gust, D.; Moore, T. A. Proc. Natl. Acad. Sci. U. S. A. 2006, 103, 53435348.

(19) Liao, P.-N.; Pillai, S.; Kloz, M.; Gust, D.; Moore, A.; Moore, T.; Kennis, J.; van Grondelle, R.; Walla, P. Photosynth. Res. 2012, 111, 237-243.

(20) Horton, P.; Johnson, M. P.; Perez-Bueno, M. L.; Kiss, A. Z.; Ruban, A. V. FEBS Lett. 2008, 275, 1069-1079.

(21) Schmid, V. Cell. Mol. Life Sci. 2008, 65, 3619-3639.

(22) Peter, G. F.; Thornber, J. P. J. Biol. Chem. 1991, 266, 1674516754.

(23) Liu, Z.; Wang, K.; Kuang, T.; Zhang, J.; Gui, L.; An, X.; Chang, W. Nature 2004, 428, 287-292.

(24) Standfuss, J.; van Scheltinga, A. C. T.; Lamborghini, M.; Kühlbrandt, W. EMBO J. 2005, 24, 919-928.

(25) Kirchhoff, H. Trends Plant Sci. 2008, 13, 201-207.

(26) van Oort, B.; van Hoek, A.; Ruban, A. V.; van Amerongen, H. FEBS Lett. 2007, 581, 3528-3532.

(27) Liao, P.-N.; Bode, S.; Wilk, L.; Hafi, N.; Walla, P. J. Chem. Phys. 2010, 373, 50-55.

(28) Haferkamp, S.; Haase, W.; Pascal, A. A.; van Amerongen, H.; Kirchhoff, H. J. Biol. Chem. 2010, 285, 17020-17028.

(29) Berthold, D. A.; Babcock, G. T.; Yokum, C. F. FEBS Lett. 1981, 134, 231-234.

(30) Porra, R. J.; Thompson, W. A.; Kriedemann, P. E. Biochim. Biophys. Acta, Bioenerg. 1989, 975, 384-394. 
(31) Haferkamp, S.; Kirchhoff, H. Photosynth. Res. 2008, 95, 129134.

(32) Kühlbrandt, W.; Thaler, T.; Wehrli, E. J. Cell. Biol. 1983, 96, $1414-1424$.

(33) Andreeva, A.; Stoitchkova, K.; Busheva, M.; Apostolova, E. J. Photochem. Photobiol. B 2003, 70, 153-162.

(34) Kirchhoff, H.; Tremmel, I.; Haase, W.; Kubitscheck, U. Biochemistry 2004, 43, 9204-9213.

(35) Mullet, J. E.; Arntzen, C. J. Biochim. Biophys. Acta 1980, 589, 100-117.

(36) Ruban, A. V.; Horton, P. Biochim. Biophys. Acta 1992, 1102, 3038.

(37) Vasil'ev, S.; Irrgang, K. D.; Schrötter, T.; Bergmann, A.; Eichler, H. J.; Renger, G. Biochemistry 1997, 36, 7503-7512.

(38) Kirchhoff, H.; Hinz, H.-J.; Rösgen, J. Bioch. Biophys. Acta, Bioenerg. 2003, 1606, 105-116.

(39) Staehelin, L. A.; Staay, G. W. M. Structure, Composition, Functional Organization and Dynamic Properties of Thylakoid Membranes. In Oxygenic Photosynthesis: The Light Reactions; Ort, D. R., Yocum, C. F., Eds.; Kluwer: Dordrecht, The Netherlands, 1996; pp $11-30$.

(40) Barros, T.; Kühlbrandt, W. Biochim. Biophys. Acta, Bioenerg. 2009, 1787, 753-772.

(41) Miloslavina, Y.; Wehner, A.; Lambrev, P. H.; Wientjes, E.; Reus, M.; Garab, G.; Croce, R.; Holzwarth, A. R. FEBS Lett. 2008, 582, $3625-3631$.

(42) Kirchhoff, H.; Mukherjee, U.; Galla, H. J. Biochemistry 2002, 41, $4872-4882$.

(43) Betterle, N.; Ballottari, M.; Zorzan, S.; de Bianchi, S.; Cazzaniga, S.; Dall'Osto, L.; Morosinotto, T.; Bassi, R. J. Biol. Chem. 2009, 284, $15255-15266$.

(44) Johnson, M. P.; Goral, T. K.; Duffy, C. D. P.; Brain, A. P. R.; Mullineaux, C. W.; Ruban, A. V. Plant Cell Online 2011, 23, 14681479.

(45) Li, X. P.; Björkman, O.; Shih, C.; Grossman, A. R.; Rosenquist, M.; Jansson, S.; Niyogi, K. K. Nature 2000, 403, 391-395. 